\title{
Simulation of an Automobile Portfolio
}

\author{
by Freddy Corlier *, Jean Lemaire * and Dunia Muhokolo **
}

\section{Objectives of the simulation}

In 1971 all the Belgian companies introduced in automobile third party liability a compulsory merit-rating system. From the very first years of application, it appeared that the system was not well balanced, since the average collected premium constantly decreased, when the effects of inflation were removed. In 1976, for instance, a large company distributed 315 millions B.F. in bonus discounts, and collected only 2 millions of malus increases, so that the average reduction of the premium reached $27.9 \%$. As this figure increases from year to year, a simulation of the portfolio of the company was performed in order to forecast the future income of this branch and to evaluate the time necessary to reach stability.

Of course the simulation presents many side advantages. For instance many results on bonus-malus systems are valid only asymptotically (Lemaire [5], Loimaranta [7], Vepsäläinen [8]). If these results are to be applied, for example to compare different systems, it is necessary to verify if the evolution of the portfolio quickly converges to a "practical equilibrium".

The idea of using simulation to study the variation of an insurance premium has already been used by Bohman [1].

\section{The Belgian merit-rating system}

Since the ministerial decree of April 1971, all the companies are compelled to apply the following system. There are 18 classes.

* Institut de Statistique of the Université Libre de Bruxelles.

** Université Nationale du Zaïre. 


\section{Table 1}

\begin{tabular}{|c|c|}
\hline Class & Premium level \\
\hline 18 & 200 \\
17 & 160 \\
16 & 140 \\
15 & 130 \\
14 & 120 \\
13 & 115 \\
12 & 110 \\
11 & 105 \\
10 & 100 \\
9 & 100 \\
8 & 95 \\
7 & 90 \\
6 & 85 \\
5 & 80 \\
4 & 75 \\
3 & 70 \\
2 & 65 \\
1 & 60 \\
\hline & \\
\hline
\end{tabular}

The sedentary drivers (the policy-holders using their cars only to and from work and for pleasure purposes) have access to the system in class 6 while the non-sedentaries must enter in class 10 . Sedentaries represent nearly $87 \%$ of the portfolio. The transition rules award a discount of one class for every claim-free year, and penalize the careless drivers by two classes for the first claim and three classes for each subsequent accident (as long as classes 1 and 18 are not exceeded, of course). One exception however restricts the application of the transition rules: if a policy-holder, after four consecutive claim-free years, still finds himself above class 10 , he is automatically brought back to this level. This restriction is unfortunate because it destroys the Markovian property of the process. One of the goals of the simulation was to compute the number of beneficiaries of this rule, in order to check for its usefulness.

\section{The model}

Classically, we supposed that the number of claims of a policy-holder is Poissondistributed,

$$
p_{k}(\lambda)=\frac{e^{-\lambda} \lambda^{k}}{k !}
$$


where $\lambda$ is a non-negative random variable admitting a $\Gamma$ distribution

$$
d U(\lambda)=\frac{\tau^{a} e^{-\tau \lambda} \lambda^{a-1}}{\Gamma(a)} d \lambda \quad a, \tau>0 .
$$

It is well known (see for instance Derron [2]) that in this case the distribution of the number of claims in the portfolio is a negative binomial

$$
p_{k}=\left(\begin{array}{c}
k+a-1 \\
k
\end{array}\right)\left(\frac{\tau}{1+\tau}\right)^{a}\left(\frac{1}{1+\tau}\right)^{k} .
$$

In Lemaire [6] we observed during one year the entire portfolio of the company. Table 2 shows that the preceding model provides a fairly good fit, accepted by the $\chi^{2}$-test of goodness of fit $(\alpha=5 \%)$.

Table 2

\begin{tabular}{|c|r|r|r|}
\hline \multirow{2}{*}{ Number of claims } & \multicolumn{3}{|c|}{ Absolute frequencies } \\
\cline { 2 - 4 } & Observed & Adjusted & Simulated \\
\hline 0 & 96,978 & $96,985.5$ & 9,713 \\
1 & 9,240 & $9,222.5$ & 909 \\
2 & 704 & 711.7 & 68 \\
3 & 43 & 50.7 & 7 \\
4 & 9 & 3.6 & 0 \\
More than 4 & 0 & 0 & 0 \\
& 106,974 & 106,974 & 10,697 \\
\hline
\end{tabular}

Mean $=.1011 \quad \tau=15.8778$

Variance $=.1074 \quad a=1.6049$

\section{The simulation technique and the program}

The major difficulty of this program is to sample from a $\Gamma$ random variable. It is sufficient to generate the distribution

$$
f(x)=\frac{x^{a-1} e^{-x}}{\Gamma(a)} \quad x \geqslant 0, a>0
$$

and to perform the transformation $\lambda=x / \tau$. If $a$ is an integer, $a \Gamma$ may be considered as a sum of $a n$ independent negative exponential variables. When the shape parameter $a$ is not an integer, however (and this will be the case in most of our examples), there is no simple method for generating $a \Gamma$. We used the following mixed procedure, following Wallace [9] and Fishman [3]'s recommendations :

- Jöhnk's algorithm when $a<1$;

- Wallace's rejection method when $1 \leqslant a<5$;

- the so-called probability switch method when $a>5$. 
As a test, we simulated a portfolio of 10,697 policies with the characteristics of the afore-mentioned observed distribution, i.e. a mean of .1011 and a variance of .1074 . The simulated distribution agrees with both the observed and the adjusted distributions (see Table 2).

\section{Results}

5.1. Using the same values of the parameters, the program was ran for a portfolio of 10,000 new sedentaries during a period of 70 years. We first observed the extremely low number of beneficiaries of the exception to the transition rules. During year 70 , for instance, only 12 policies took advantage of the restriction to regain class 10 ( 9 from class 15, 2 from class 12,1 from class 13 and none from class 14). This costed the company $.05 \%$ of its income. Over the entire period, the average annual number of beneficiaries was 9.24. This naturally casts some doubts on the efficiency of the restriction. From a theoretical point of view, it really was not worthwhile to render the process non-Markovian in order to distribute a discount to very few people.

Fig. 1 (curve a) presents the evolution of the mean premium (assuming that the commercial premium at level 100 is 10,000 B.F.). One notices a steep decrease of the income of the company during the first five years, the time taken by the best drivers to reach the minimal class. After that, the income continues to decrease more slowly, but irregularly. Although the process is not periodic in the Markovian sense of the word, there is a noticeable period of three years in the distribution of the policies among the classes, and, to a lesser extent, in the mean premium : one observes "waves" of policies arriving in class 1 every three years; this is of course due to the fact that it takes two claim-free years to return to the original level after an accident. The waves gradually fade out and the premium stabilizes after 12 to 15 years; the premium only decreases by 12 B.F. between years 16 and 70, with oscillations never exceeding 9 B.F. One can thus consider that practically the equilibrium state is attained after 15 years.

The evolution of the premium drastically demonstrates that the system is out of balance, since the asymptotic premium is $35.6 \%$ below the flat commercial premium at level 100 , and $24.3 \%$ below the class 6 level.

5.2. The preceding results were obtained with a static portfolio of sedentaries, all entering the system at the same moment. We have then slightly altered the program in order to be more realistic: we have introduced $13 \%$ of non-sedentaries, and taken into account the fact that every year $.93 \%$ of the policy-holders quit the company while $7.17 \%$ new policies are registered. The conclusions concerning the non-Markovian restriction are not affected but the premium takes a few more years to stabilize. The oscillations of the mean premium (Fig. 1, curve b) are smaller. One notices that the constant flow of the new policies keeps the premium level above the premium of a static portfolio. So the new policy-holders are penalized and "pay for the others" for a few years. The asymptotic premium is $33.5 \%$ below the premium at level 100 , $23 \%$ below the premium at year 0 . It is interesting to note the extremely good agreement between the mean simulated (7199.5) and observed (7209.6) premiums.

5.3. Naturally the preceding results are strongly influenced by the choice of the parameters of the $\Gamma$-distribution. Therefore we have computed the number of benefi- 
ciaries of the restriction (Table 3 ) and the mean premium after 30 years (Table 4) for a portfolio of 1,000 policy-holders (sedentaries and non-sedentaries) for different values of the mean and the variance of the distribution of the number of claims. The evolution of the premium is shown on Fig. 1 (curves $c$ and d) for two particular cases. The small number of cases per class explains for some oddities in Table 3 (see for instance the first line).

Table 3

\begin{tabular}{|r|rrrrrrrrrrr|}
\hline \multicolumn{1}{r|}{$\mathrm{m}$} & .10 & .12 & .14 & .16 & .18 & .20 & .22 & .24 & .26 & .28 & .30 \\
\hline .13 & 1 & 1 & & & & & & & & & \\
.16 & 2 & 3 & 3 & & & & & & & & \\
.19 & 2 & 5 & 6 & 3 & 2 & & & & & & \\
.22 & 1 & 1 & 3 & 5 & 5 & 3 & & & & & \\
.25 & 1 & 1 & 3 & 4 & 7 & 9 & 4 & 8 & & & \\
.28 & 2 & 5 & 2 & 3 & 5 & 6 & 10 & 3 & 7 & & \\
.31 & 0 & 0 & 2 & 2 & 3 & 9 & 5 & 8 & 7 & 14 & 13 \\
.34 & 3 & 0 & 2 & 3 & 7 & 5 & 4 & 9 & 11 & 9 & 14 \\
.37 & 0 & 1 & 2 & 1 & 4 & 5 & 5 & 4 & 4 & 8 & 14 \\
.40 & 1 & 1 & 2 & 2 & 2 & 8 & 4 & 2 & 3 & 9 & 11 \\
\hline
\end{tabular}

Table 4

\begin{tabular}{|c|c|c|c|c|c|c|c|c|c|c|c|}
\hline & .10 & .12 & .14 & .16 & .18 & .20 & .22 & .24 & .26 & .28 & .30 \\
\hline .13 & 6,865 & 6,692 & & & & & & & & & \\
\hline .16 & 6,907 & 6,915 & 7,041 & & & & & & & & \\
\hline .19 & 7,046 & 7,095 & 7,255 & 7,279 & 7,132 & & & & & & \\
\hline .22 & 6,760 & 6,999 & 7,248 & 7,511 & 7,706 & 7,531 & & & & & \\
\hline .25 & 6,850 & 7,249 & 7,248 & 7,591 & 7,894 & 7,875 & 8,032 & 7,904 & & & \\
\hline .28 & 6,749 & 7,045 & 7,247 & 7,557 & 7,905 & 7,908 & 8,190 & 8,226 & 8,290 & & \\
\hline .31 & 6,795 & 7,026 & 7,336 & 7,474 & 7,662 & 7,930 & 8,138 & 8,620 & 8,656 & 8,684 & 8,902 \\
\hline .34 & 6,748 & 7,082 & 6,936 & 7,518 & 7,661 & 8,271 & 8,294 & 8,612 & 8,650 & 8,776 & 8,950 \\
\hline .37 & 6,537 & 6,923 & 7,132 & 7,469 & 7,529 & 7,820 & 7,989 & 8,287 & 8,458 & 8,955 & 9,280 \\
\hline .40 & 6,701 & 6,708 & 7,205 & 7,214 & 7,420 & 7,608 & 7,967 & 8,187 & 8,923 & 8,652 & 9,056 \\
\hline
\end{tabular}

The number of beneficiaries increases with the claim frequency $m$, but remains in any case very low. The stabilization time does not seem to be much affected by the variation of the parameters. The system is financially balanced only for very high and uncommon values of the claim frequency (.28 and .30$)$. 


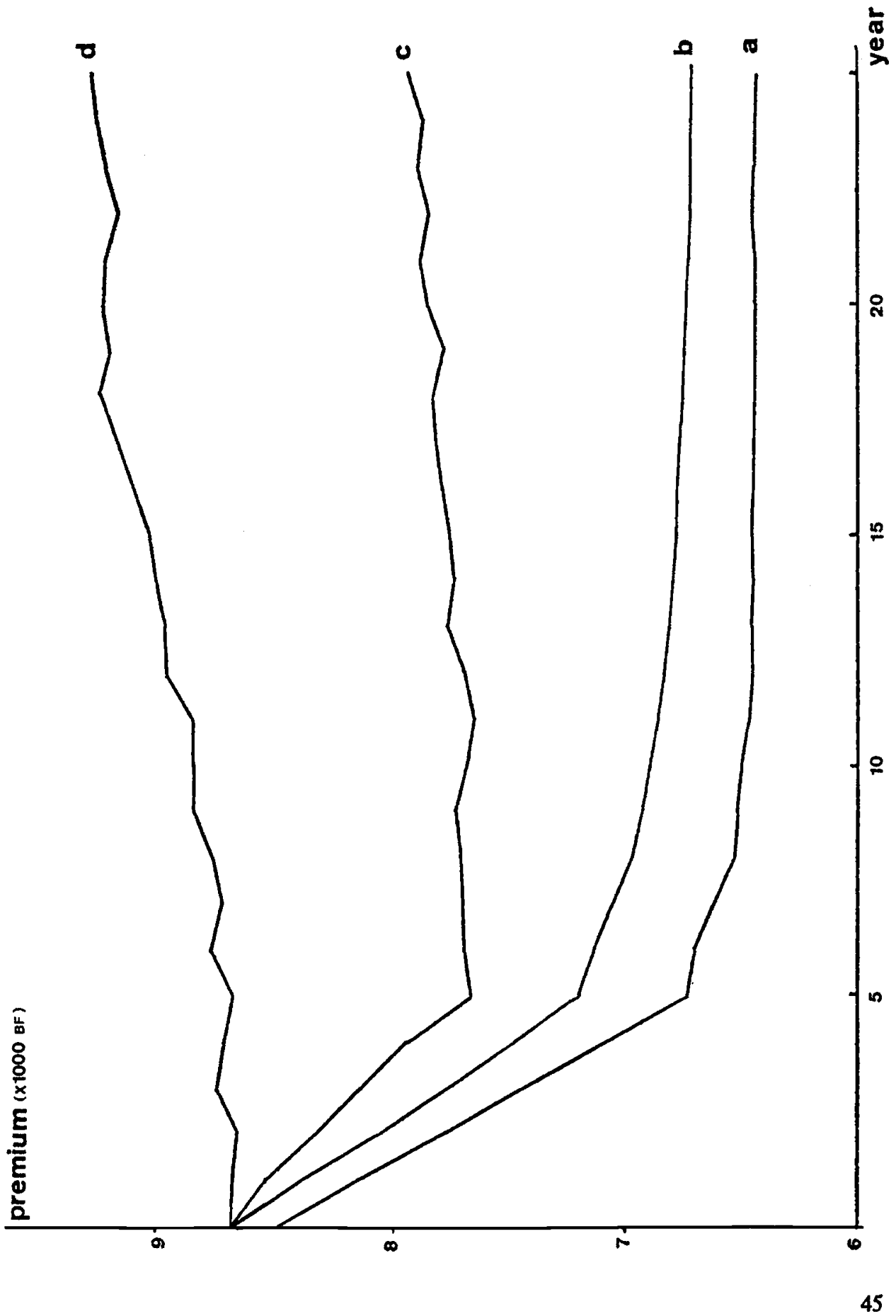


With some discrepancies due to the rather weak number of policies, the premium increases linearly with the claim frequency. For $\sigma^{2}=.31$, for instance, the equation of the line of best fit is

$$
\text { Premium }=10,863 m+5,757 \text {, }
$$

with a correlation coefficient of .9898 . The premium thus increases on the average by 1,068 B.F. for each augmentation of .1 of the claim frequency. As many authors have already pointed out (Lemaire [4] and [5], Loimaranta [7], Vepsäläinen [8]), this bonus-malus system (like many others in Europe) is unefficient; since the premium should double every time the claim frequency does, the slope of the regression line should be 39,647 instead of 10,681 . We can thus estimate the overall efficiency of the system by

$$
\frac{10,861}{39,647}=27.32 \%
$$

Note that this efficiency is unweighted in the sense that each value of $\bar{m}$ has the same power; an efficiency concept weighted by a structure function would supply a lower value.

5.4. The simulation does not take into account the so-called " hunger for bonus". Lemaire [5] has computed the optimal strategy of the policy-holder for every value of the claim frequency. Inserting his algorithm into the program allows us to use the number of declared claims (instead of the number of occurred claims) by comparing the probability that a policy-holder defrays himself the costs of a claim to a random number. Since this comparison has to be performed for every accident of each policyholder (and this implies the calculation of the optimal strategy) the computer time quickly becomes excessive. We were only able to simulate a portfolio of 100 policies (with the characteristics of 5.1) for 30 years. After a few years, most of the policies find themselves in classes 1,2 or 3 and the average premium drops nearly to the minimal level of 6,000 B.F.

\section{REFERENCES}

1. BOHMAN, H.: "On the use of simulation technique", Scandinavian Actuarial Journal, 1974, 175-178.

2. DERRON, M.: "Mathematische Probleme der Automobilversicherung", Mitteilungen der Vereinigung Schweizerischer Versicherungsmathematiker, 1962, 103-123.

3. FISHMAN, G. : "Sampling from the gamma distribution on a computer", Comm. A.C.M., 1976, 407-409.

4. LEMAIRE, J.: "Driver versus company: optimal behaviour of the policy-holder", Scandinavian Actuarial Journal, 1976, 209-219.

5. LEMAIRE, J. : "La soif du bonus", The ASTIN Bulletin, Vol. IX, 1977, 181-190.

6. LEMAIRE, J.: "Selection procedures of regression analysis applied to automobile insurance", Mitteilungen der Vereinigung Schweizerischer Versicherungsmathematiker, 1977, 143-160.

7. LOIMARANTA, K.: "Some asymptotic properties of bonus systems", The ASTIN Bulletin, Vol. VI, 1972, 233-245.

8. VEPSALAINEN, S. : "Applications to a theory of bonus systems", The ASTIN Bulletin, Vol. VI, 1972, 212-221.

9. WALLACE, N.: "Computer generation of gamma random variates with non-integral shape parameters, Comm. A.C.M., 1974, 691-695. 